Case Report

\title{
Ventilator Management of Bronchopleural Fistula Secondary to Methicillin-Resistant Staphylococcus aureus Necrotizing Pneumonia in a Pregnant Patient with Systemic Lupus Erythematosus
}

\author{
Ahmed F. Alohali, Saleh Abu-Daff, Kamardeen Alao, and Mohammed Almaani \\ Department of Pulmonary and Critical Care, King Fahad Medical City, P.O. Box 59046, Riyadh 11525, Saudi Arabia \\ Correspondence should be addressed to Ahmed F. Alohali; dr.aalohali@gmail.com
}

Received 11 December 2016; Revised 3 March 2017; Accepted 12 March 2017; Published 14 May 2017

Academic Editor: John Kortbeek

Copyright (C) 2017 Ahmed F. Alohali et al. This is an open access article distributed under the Creative Commons Attribution License, which permits unrestricted use, distribution, and reproduction in any medium, provided the original work is properly cited.

\begin{abstract}
Managing mechanical ventilation in patient with bronchopleural fistula with coexisting acute respiratory distress syndrome is a challenging situation for the intensivist. We are reporting a case of a pregnant patient with systemic lupus erythematosus on immunosuppressive medications who developed methicillin-resistant Staphylococcus aureus necrotizing pneumonia complicated by bronchopleural fistula and acute respiratory distress syndrome.
\end{abstract}

\section{Introduction}

Bronchopleural fistula (BPF) is an infrequently encountered complication in intensive care units (ICU) in patients who have not undergone thoracic surgery [1] and poses difficulty in ventilator management. Coexisting acute respiratory distress syndrome (ARDS) adds to this challenge. We here report a case of a pregnant woman known to have systemic lupus erythematosus (SLE) who developed BPF as a consequence of methicillin-resistant Staphylococcus aureus (MRSA) necrotizing pneumonia, after which she developed ARDS.

\section{Case Presentation}

A 24-year-old 16-week pregnant woman presented at the emergency department (ED) with fever and productive cough of yellowish sputum of 3-week duration. She is a known case of SLE and was receiving prednisolone $(40 \mathrm{mg}$ daily), azathioprine (50 mg twice daily), and hydroxychloroquine (200 $\mathrm{mg}$ twice daily).

On physical examination, she was febrile with a temperature of $38.4^{\circ} \mathrm{C}$. However, oxygen saturation was maintained at $96 \%$ in room air, with decreased breath sounds on the right infrascapular area.
Her chest roentgenogram on presentation (Figure 1) showed a right upper lobe cavitary lesion, with a large right pneumothorax and pleural effusion. A right-sided chest tube was inserted, which drained pus. Chest computed tomography (CT) (Figure 2) revealed multiple bilateral pulmonary cavitation (more on the right), moderate right-sided pleural effusion, air-space infiltrates, and a right-sided cavitarypleural communication.

She was admitted to the hospital and antibiotic treatment was commenced. Her sputum and pleural fluid cultures were positive for MRSA. Subsequently, azathioprine was stopped and prednisolone was tapered off. Fetal ultrasound showed a single viable fetus with normal biometrics.

During her hospital stay, she developed shortness of breath and a decrease in oxygen saturation, which required mechanical ventilation (MV) and ICU admission. Her chest roentgenogram (Figure 3) showed multiple air-filled cavitary right lung lesions, right-sided pleural effusion, and progression of the air-space disease in the left lung.

In the ICU, she was fully sedated and paralyzed, and her blood pressure was supported by means of vasopressors. Her initial mode of MV was pressure control ventilation (PCV) with inspiratory pressure of $34 \mathrm{cmH} 2 \mathrm{O}$, positive endexpiratory pressure (PEEP) of $10 \mathrm{cmH} 2 \mathrm{O}$, ventilator rate of 
TABLE 1: Blood gas change on different modes of mechanical ventilation.

\begin{tabular}{lccccc}
\hline Mode of MV & PH & PCo2 & PO2 & HCO3 & Sat. \\
\hline PCV & 7.05 & 85 & 40 & 23 & 53 \\
HFOV & 7.09 & 68 & 79 & 20 & 90 \\
CMV 1 & 7.35 & 45 & 55 & 24 & 86 \\
DLV & 7.47 & 36 & 71 & 26 & 95 \\
CMV 2 & 7.44 & 45 & 138 & 30 & 98 \\
\hline
\end{tabular}

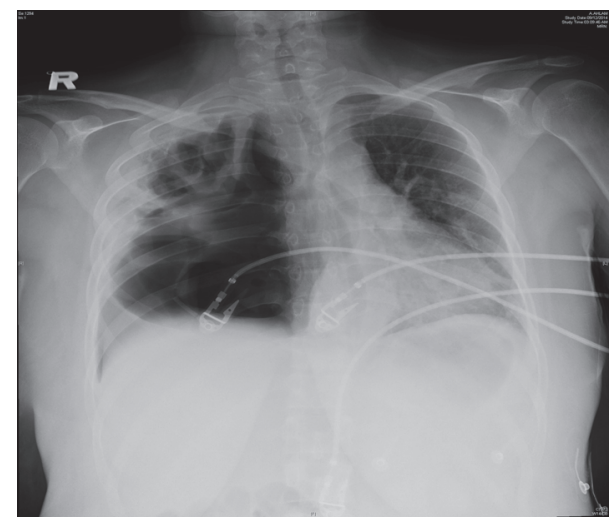

Figure 1: Chest X-ray showing right upper lobe cavitary lesion with a large pneumothorax and pleural effusion.

$22 / \mathrm{min}$, and fractional inspired oxygen concentration $\left(\mathrm{FiO}_{2}\right)$ of $100 \%$. After initiation of MV, the air leak through the right BPF worsened considerably. Her arterial blood gas (ABG) after initiation of MV is displayed in Table 1.

Due to sustained hypoxemia, nitric oxide (NO) was added to her MV, which was changed to high frequency oscillatory ventilation (HFOV) with a frequency of $4.5 \mathrm{~Hz}$, amplitude of $70 \mathrm{cmH}_{2} \mathrm{O}$, mean air way pressure of $26 \mathrm{cmH}_{2} \mathrm{O}$, and $\mathrm{FiO}_{2}$ of $100 \%$. Her gas exchange improved gradually over a few days, she was weaned off NO, and her oxygen requirements decreased. HFOV was switched to controlled mandatory ventilation (CMV) with a tidal volume (TV) of $450 \mathrm{~mL}$, rate of 28/min, $\mathrm{PEEP}$ of $12 \mathrm{cmH}_{2} \mathrm{O}$, and $\mathrm{FiO}_{2} 45 \%$ ABG in Table 1.

Due to the persistent large air leak from the right lung, however, it was decided to initiate differential lung ventilation (DLV). The settings were as follows: left lung CMV TV $200 \mathrm{~mL}$, PEEP $8 \mathrm{cmH} 2 \mathrm{O}$, rate $12 / \mathrm{min}$, and $\mathrm{FiO}_{2} 35 \%$; right lung PCV pressure $15 \mathrm{cmH} 2 \mathrm{O}$, PEEP $4 \mathrm{cmH} 2 \mathrm{O}$, rate $12 / \mathrm{min}$, and $\mathrm{FiO}_{2} 60 \%$. The $\mathrm{ABG}$ obtained by these means is shown in Table 1. Air leak improved gradually over 11 days. DLV was switched to CMV with a TV of $300 \mathrm{~mL}$, rate of $14 / \mathrm{min}$, PEEP of $4 \mathrm{cmH} 2 \mathrm{O}$, and $\mathrm{FiO} 235 \%$, and $\mathrm{ABG}$ was as summarized in Table 1.

The patient was ultimately weaned off MV and subsequently extubated. She was transferred to the ward on nasal cannula, where she gradually improved, and was later discharged home. Her chest roentgenogram and CT are shown in Figures 4 and 5.
TABLE 2

Pulmonary resection

Persistent spontaneous pneumothorax, including ruptured bulla(e)

Necrotizing pulmonary infection

Inflammatory lung diseases

Malignancy

After chemotherapy or radiotherapy for lung cancer

Thoracic trauma

After lung transplant

ARDS

Iatrogenic (e.g., chest tube insertion and central venous line

placements)

Broncholithiasis

Idiopathic

\section{Discussion}

$\mathrm{BPF}$ is a direct communication between the airway and the pleural cavity. It can present acutely as a pneumothorax or subacutely or chronically as empyema [1]. Pneumonectomy and lobectomy are the most common causes of BPF, with reported incidence rates between $4.5 \%$ and $20 \%$ after pneumonectomy and $0.5 \%$ after lobectomy [2]. Other causes of BPF are listed in Table 2 [3]. The reported mortality of BPF is between $18 \%$ and $50 \%$, with the most common causes of death being tension pneumothorax and aspiration pneumonia [4]. BPF leads to an air leak which, if significant, compromises pulmonary gas exchange, leading to respiratory acidosis and severe hypoxemia.

The management of BPF is supportive and can also involve MV and interventional management. Patients may require chest tube insertion to drain the pneumothorax and empyema. The chest tube should preferably be wide and short to minimize the flow of air through the fistula and facilitate drainage of the empyema [4] and should be used together with antibiotic management.

If the patient requires mechanical ventilation, the strategies for MV rely on minimizing the air leak and maintaining acceptable gas exchange. These can be achieved by reducing the airway pressure through the use of low TV ventilation, lowering the PEEP, shortening the inspiratory time, and reducing the respiratory rate [3]. There are no reported controlled studies comparing the various modes of conventional MV in the setting of BPF. Pierson et al. reported a case series of 39 patients with BPF maintained on conventional ventilation and showed significant mortality in patients with air leak greater than $500 \mathrm{~mL} /$ breath, as well as in those developing BPF late in the course of their illness.

Only two of the patients in this series developed significant respiratory acidosis that was unresponsive to conventional ventilation, despite the presence of major air leaks [5]. Litmanovitch et al. reported one case in which pressure control ventilation was successfully used in a patient with coexisting ARDS [6].

There are several reports of successful use of both high frequency jet ventilation (HFJV) and HFOV (3) in the setting 

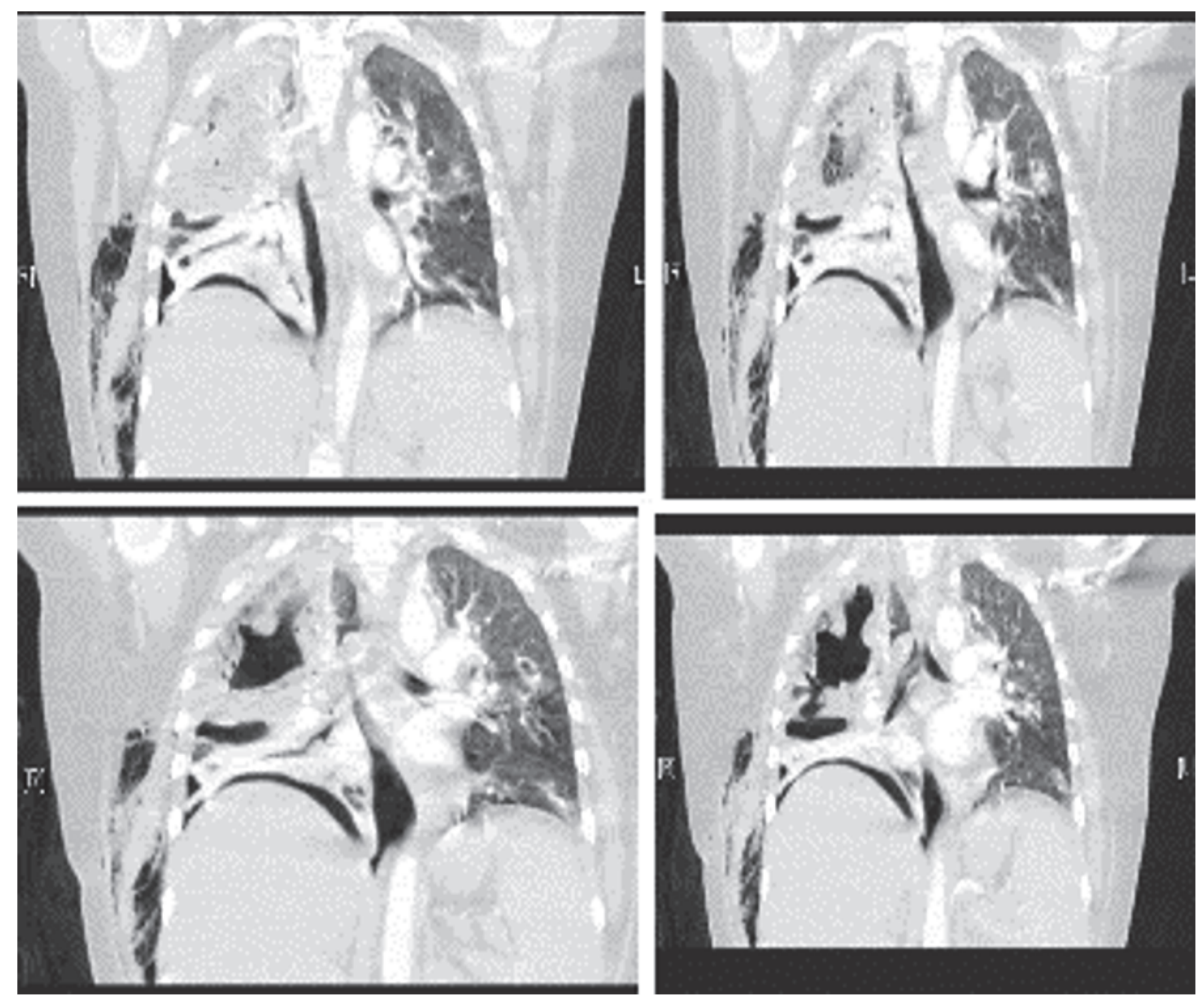

Figure 2: Chest computed tomography revealed multiple bilateral cavitation (more on the right), moderate right-sided pleural effusion, air-space infiltrates, and a right-sided cavitary-pleural communication.

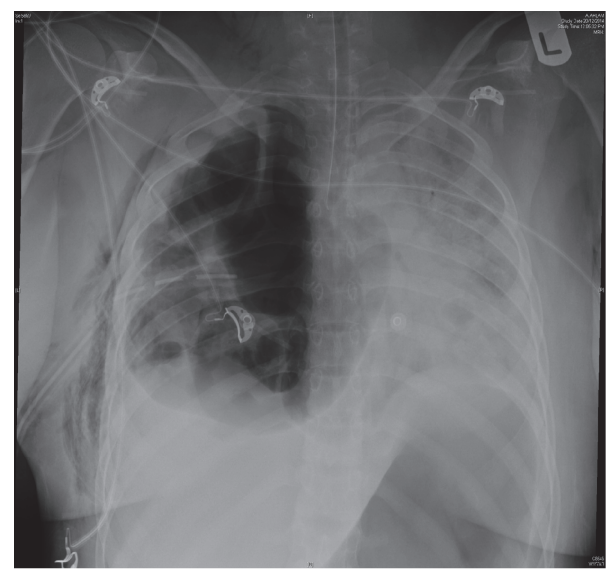

FIGURE 3: Chest X-ray showing multiple air-filled cavitary right lung lesions, right-sided pleural effusion, and progression of the air-space disease in the left lung.

of BPF. HFJV generally seems to be useful in patients with proximal BPF, without significant underlying lung disease. HFOV may be more suitable for patients with high-output BPF with poor lung compliance [4].

On the other hand, DLV facilitates optimal ventilation of the normal or less-diseased lung, while allowing maintenance of lower airway pressures on the affected side by reducing the air leak. No controlled studies and guidelines for the use of DLV in BPF are currently available. Only case reports of

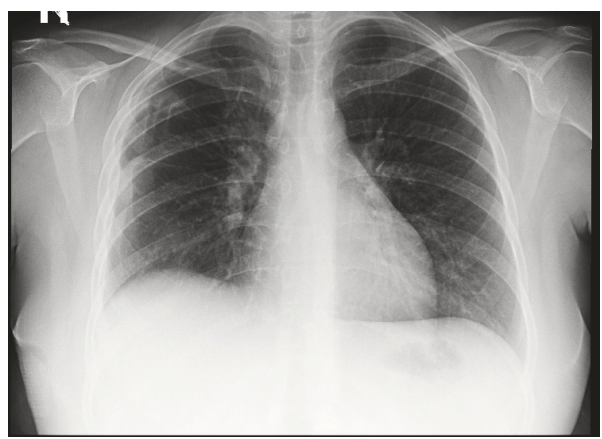

Figure 4: Chest X-ray on discharge showing resolution of the bilateral lung infiltrates, pleural effusions, and surgical emphysema.

the use of DLV in the setting of BPF, with different modes of ventilation for each lung, are currently available in the medical literature. Cinnella et al. reported successful weaning of a case of lung contusion from DLV to conventional MV once the compliance difference became less than 20\% [7].

Extracorporeal membrane oxygenation ECMO is a rapidly evolving therapy used in the management of refractory respiratory failure. There are many case report and series that reported the successful use of ECMO in cases with BPF. Daoud et al. reported successful use of arteriovenous ECMO in 5 patients via femorofemoral cannulation in the setting refractory respiratory failure associated with bronchial fistula and acute lung injury after thoracic operations and failure of 


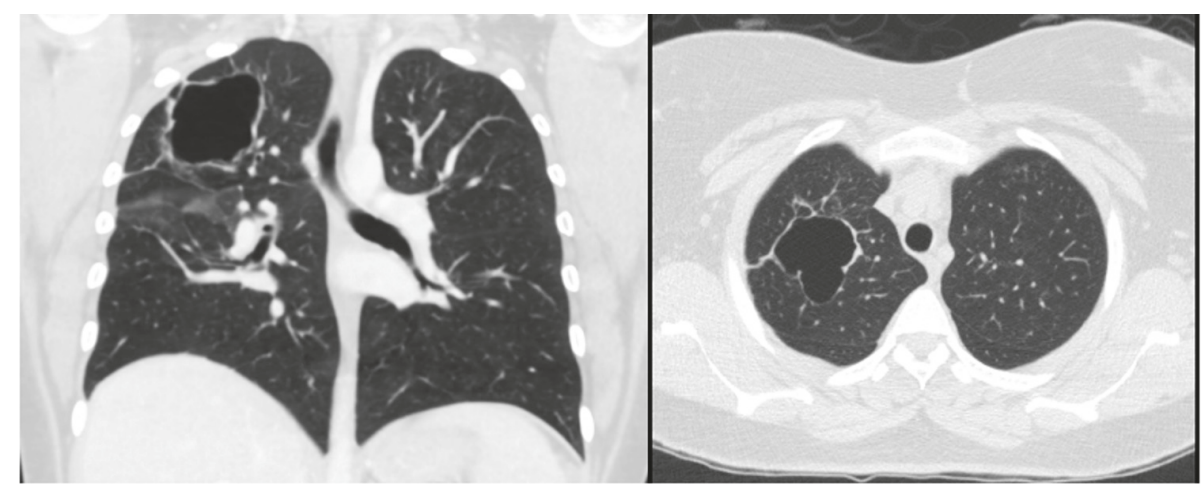

FIGURE 5: Chest CT on discharge showing resolution of the BPF and development of thin wall right upper lobe cavity.

conventional ventilation [8]. In a case series of 2 pediatric patients with severe Staphylococcus aureus-induced necrotizing, both patients were temporarily placed on venoarterial ECMO support and subsequently survived the infection [9]. In another case report, a 22-year-old female with necrotizing pneumonia and refractory hypoxemia was placed on venovenous ECMO support to minimize the risk of acute lung injury, particularly to the lesser affected lung (right). She was subsequently decannulated and had a left pneumonectomy with a favorable outcome [10]. Garlick et al. reported a successful management of 16-year-old boy posttraumatic $\mathrm{BPF}$ resistant to conventional ventilation with differential lung ventilation and ECMO via bicaval dual lumen catheter [11]. ECMO support can be considered for cases of complicated necrotizing pneumonia, or necrotizing pneumonia with refractory hypoxia, by itself, or with planned surgical intervention at a later date based on available equipment, medications, and personnel experience. Larger randomized studies in this regard will be helpful to further delineate the timing of ECMO, the type, the patient population in which the most favorable outcomes can be achieved. A potential limiting factor in this approach is the use of systemic anticoagulation.

In terms of definitive treatment, aggressive infection treatment and nutritional support are essential part in surgical management. Definitive surgical treatment varies according to the cause of the BPF. Most BPFs repaired surgically are in the setting of pneumonectomy, lobectomy, and wedge resection. Buttress the suture closure preferably with vascularized omental flap, muscle, or pleural flaps if the omental flaps cannot be used [12]. In critically ill patients who will not tolerate surgery especially with proximal well visualized fistula via bronchoscope, deployment of different types of sealants and valves is prescribed in the literature as case reports $[1,3]$.

A review of the literature revealed one report of a 32-week pregnant patient who presented with respiratory failure and septic shock secondary to a ruptured pulmonary hydatid cyst, with superimposed pulmonary tuberculosis. This patient underwent thoracotomy and cesarean section and was subsequently extubated. After procedure, she developed BPF, which was treated with chest tube insertion for 3 weeks and antimicrobials [9].
The patient presented here was unique as she had been diagnosed with SLE and was on immune suppressive medication, was pregnant, and developed BPF and ARDS, and required $\mathrm{MV}$, which added to the complexity of her management. To the best of our knowledge, this is the first report of such a case.

\section{Disclosure}

This case is accepted and presented as a poster in Canada Critical Care Forum October 2015. http://cccf.multilearning .com/cccf/2015/eposter/114755/ahmed.alohali.ventilator.management.of.bronchopleural.fistula.secondary.to.html, http:// www.criticalcarecanada.com/wp-content/uploads/2015/10/ CCCF-2015-ePoster-Sessions_FINAL.pdf.

\section{Conflicts of Interest}

The authors declare that there are no conflicts of interest regarding the publication of this case report.

\section{References}

[1] J. E. McManigle, G. L. Fletcher, and M. F. Tenholder, "Bronchoscopy in the management of bronchopleural fistula," Chest, vol. 97, no. 5, pp. 1235-1238, 1990.

[2] R. J. Cerfolio, "The incidence, etiology, and prevention of postresectional bronchopleural fistula," Seminars in Thoracic and Cardiovascular Surgery, vol. 13, no. 1, pp. 3-7, 2001.

[3] K. Shekar, C. Foot, J. Fraser, M. Ziegenfuss, P. Hopkins, and M. Windsor, "Bronchopleural fistula: an update for intensivists," Journal of Critical Care, vol. 25, no. 1, pp. 47-55, 2010.

[4] M. H. Baumann and S. A. Sahn, "Medical management and therapy of bronchopleural fistulas in the mechanically ventilated patient," Chest, vol. 97, no. 3, pp. 721-728, 1990.

[5] D. J. Pierson, C. A. Horton, and P. W. Bates, "Persistent bronchopleural air leak during mechanical ventilation. A review of 39 cases," Chest, vol. 90, no. 3, pp. 321-323, 1986.

[6] M. Litmanovitch, G. M. Joynt, P. J. F. Cooper, and P. Kraus, "Persistent bronchopleural fistula in a patient with adult respiratory distress syndrome: treatment with pressure-controlled ventilation," Chest, vol. 104, no. 6, pp. 1901-1902, 1993. 
[7] G. Cinnella, M. Dambrosio, N. Brienza et al., "Independent lung ventilation in patients with unilateral pulmonary contusion. Monitoring with compliance and EtCO2," Intensive Care Medicine, vol. 27, no. 12, pp. 1860-1867, 2001.

[8] O. Daoud, P. Augustin, P. Mordant et al., "Extracorporeal membrane oxygenation in 5 patients with bronchial fistula with severe acute lung injury," Annals of Thoracic Surgery, vol. 92, no. 1, pp. 327-330, 2011.

[9] M. H. Stroud, R. Okhuysen-Cawley, R. Jaquiss, A. Berlinski, and R. T. Fiser, "Successful use of extracorporeal membrane oxygenation in severe necrotizing pneumonia caused by Staphylococcus aureus," Pediatric Critical Care Medicine, vol. 8, no. 3, pp. 282-287, 2007.

[10] T. S. Panchabhai, J. E. Khabbaza, S. Raja, A. C. Mehta, and U. Hatipoglu, "Extracorporeal membrane oxygenation and toilet bronchoscopy as a bridge to pneumonectomy in severe community-acquired methicillin-resistant Staphylococcus aureus pneumonia," Annals of Thoracic Medicine, vol. 10, no. 4, pp. 292294, 2015.

[11] J. Garlick, T. Maxson, M. Imamura, J. Green, and P. Prodhan, "Differential lung ventilation and venovenous extracorporeal membrane oxygenation for traumatic bronchopleural fistula," Annals of Thoracic Surgery, vol. 96, no. 5, pp. 1859-1860, 2013.

[12] J. D. Puskas, D. J. Mathisen, H. C. Grillo, J. C. Wain, C. D. Wright, and A. C. Moncure, "Treatment strategies for bronchopleural fistula," The Journal of Thoracic and Cardiovascular Surgery, vol. 109, no. 5, pp. 989-996, 1995. 


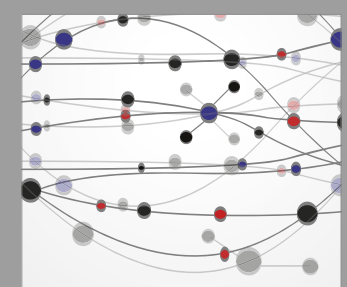

The Scientific World Journal
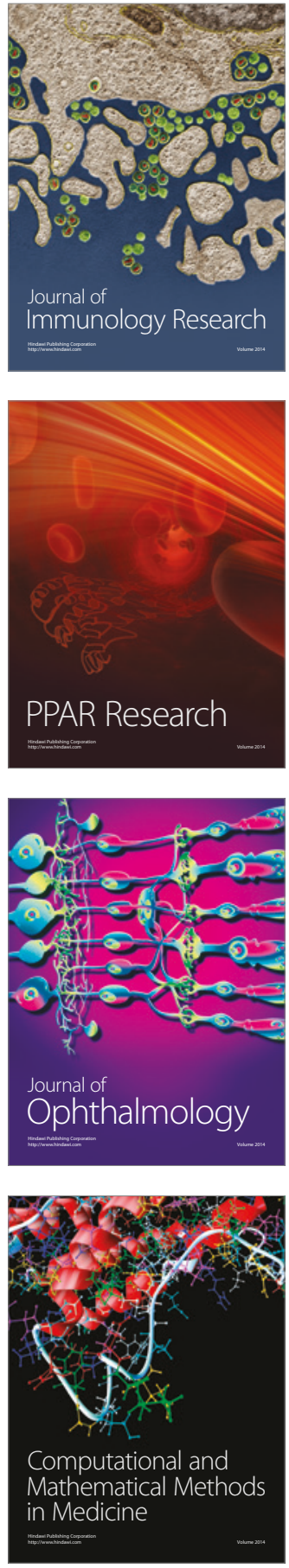

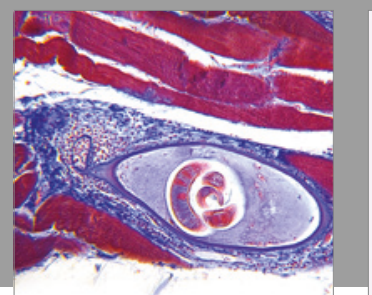

Gastroenterology Research and Practice
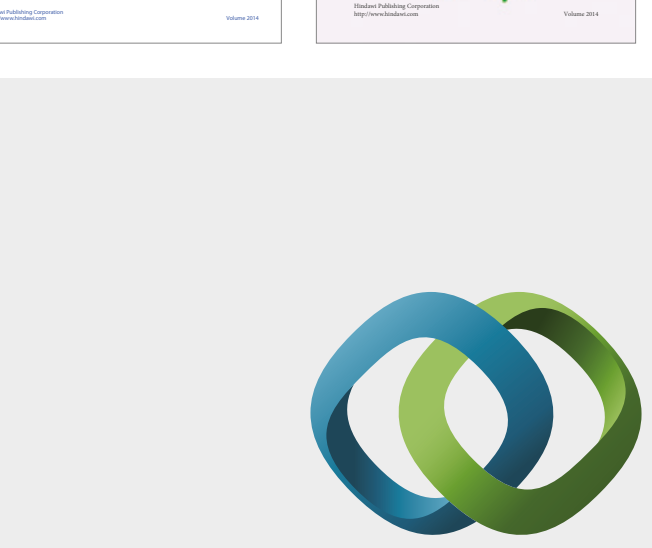

\section{Hindawi}

Submit your manuscripts at

https://www.hindawi.com
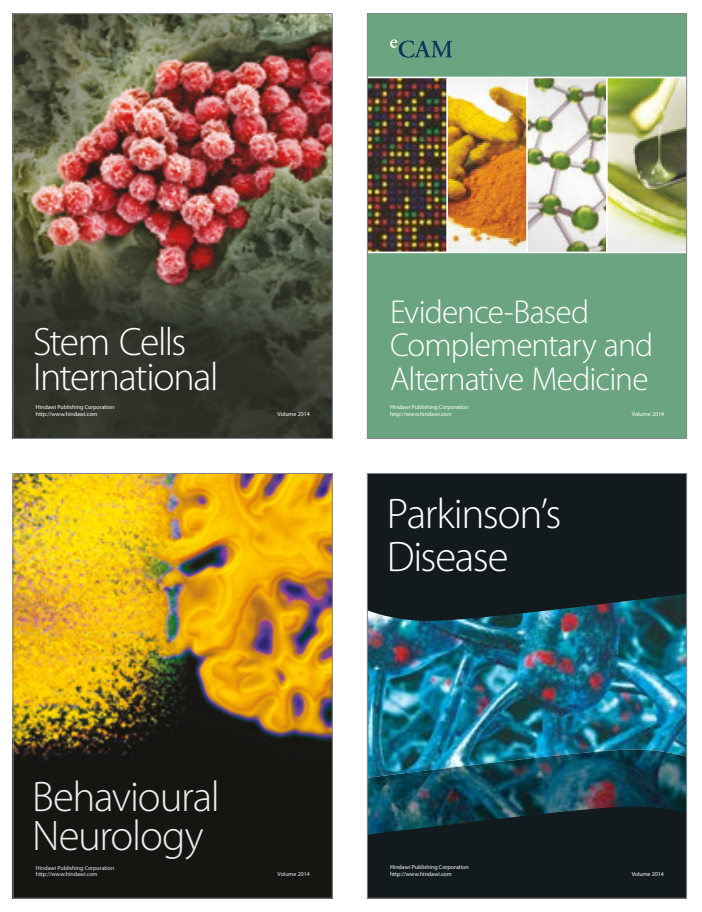
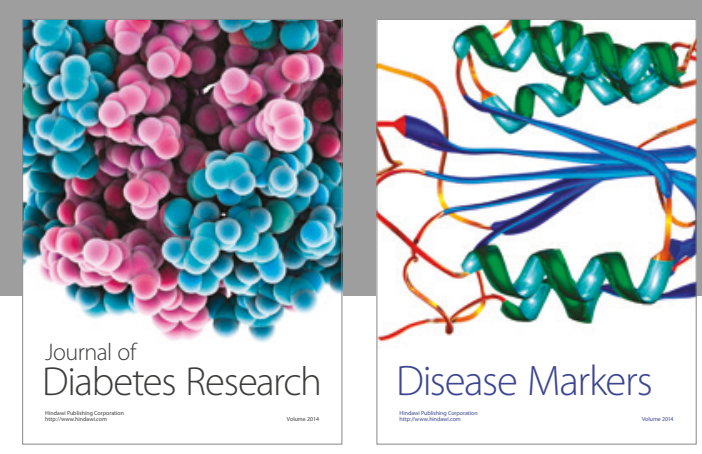

Disease Markers
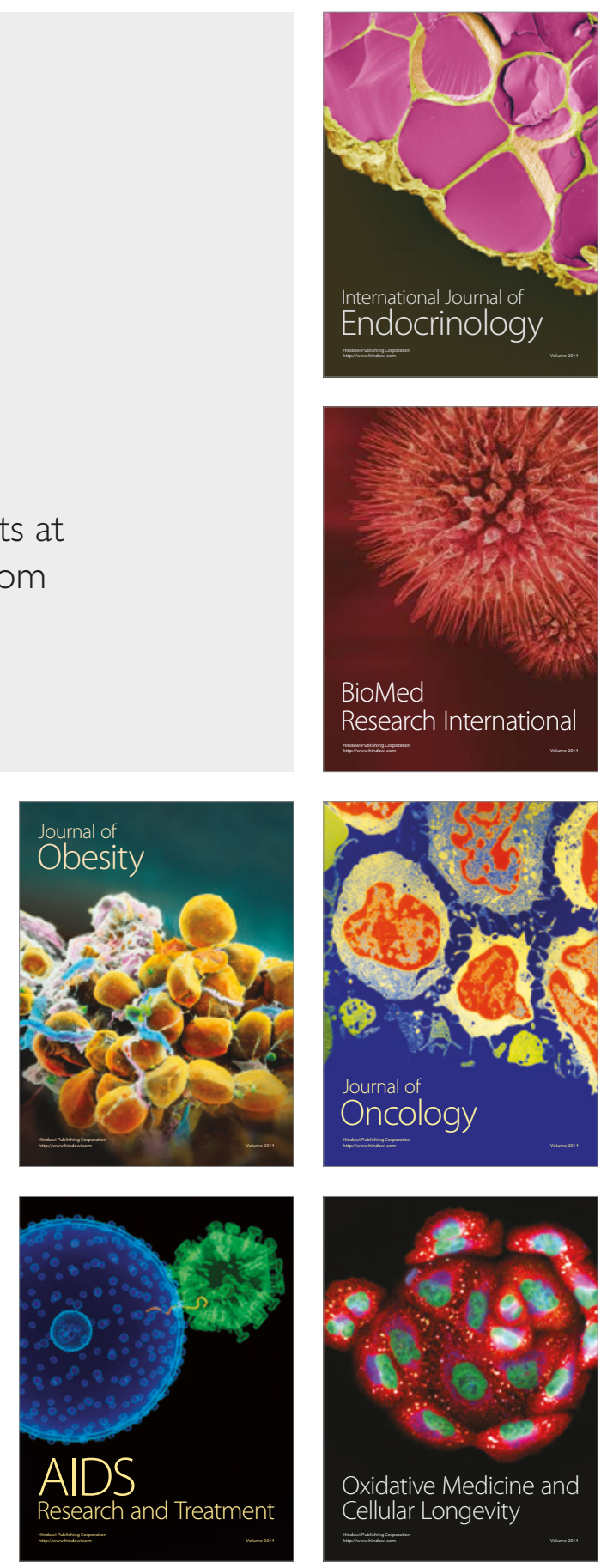